\title{
Diseño, implementación y evaluación de prácticas pedagógicas en un programa de Licenciatura con énfasis en inglés ${ }^{1}$
}

\section{Design, implementation and evaluation of teaching practices in a Bachelor program with emphasis in English}

\author{
Pilar Esther Méndez ${ }^{2}$ \\ Ximena Bonilla ${ }^{3}$
}

\begin{abstract}
Citation/ Para citar este artículo: Méndez, P. E Bonilla, X. (2016). Diseño, implementación y evaluación de prácticas pedagógicas en un programa de Licenciatura con énfasis en inglés. Colomb. Appl. Linguist. J., 18(2), pp. 49-66.

Received: 12-Feb-2015 / Accepted: 19-Sept-2015

DOI: http://dx.doi.org/10.14483/calj.v18n2.8197

\section{Resumen}

Se identifican los desafíos de una integración disciplinar en los espacios dedicados a la práctica docente de un programa de licenciatura que declara la interdisciplinariedad como enfoque curricular. Mediante el seguimiento de un modelo de investigación acción mixto y sustentado en la teoría de autoevaluación curricular (Palos, 2000 Posner, 2004; Martinello \& Cook, 2000), el diagnóstico revela la falta de articulación entre teoría y práctica. El proceso de la investigación permite, por un lado, valorar cómo profesores, estudiantes y administrativos asumen la interdisciplinariedad en el proceso de formación docente $y$, por otro, construir como resultado unos lineamientos interdisciplinarios orientados a favorecer las relaciones entre campos de formación para dar coherencia y validez a los núcleos problémicos. Los resultados sugieren que las intervenciones curriculares posteriores basadas en la implementación de lineamientos interdisciplinarios ofrecen un marco de participación más activo de docentes y estudiantes para la autoevaluación y solución de problemas específicos.
\end{abstract}

Palabras clave: currículo interdisciplinario, lineamientos, interdisciplinariedad, currículo problémico y práctica docente.

\footnotetext{
Abstract

This article identifies the challenges lived in an attempt to a disciplinary integration in the subjects dedicated to teaching practice of a Bachelor of Arts in Education. This B.A. program declares an interdisciplinary curriculum. Through a model of mixed action research, supported on the theory of curriculum evaluation (Palos, 2000; Posner, 2004; Martinello y Cook, 2000), the diagnosis reveals a lack of articulation between theory and practice; a break- up between what is declared in the official curriculum towards the interdisciplinary relationships between fields of training and what happens in practice. That is, a disconnection on the horizons of training in teaching practices and problems in

1 Artículo resultado de Proyecto de Investigación culminado, financiado por el Centro de Investigaciones y Desarrollo Científico de la Universidad Distrital Francisco José de Caldas; contrato O65 de 2008, acta de inicio febrero de 2008 y acta de cierre y liquidación de abril de 2014.

2 Universidad Distrital Francisco José de Caldas, Bogotá, Colombia. pilarmendezr@hotmail.com

3 Universidad Distrital Francisco José de Caldas, Bogotá, Colombia. ximenabvonilla@gmail.com
} 
its direction and guidance. The research process allows, on the one hand, assessing information about how teachers, students and administrative assume interdisciplinarity in the process of teacher education. On the other hand, the process permitted to provide some guidelines aimed at encouraging interdisciplinary relationships among fields of training as a result. Those guidelines attempt to give coherence and validity to the problematic nucleus. Findings suggest that curriculum interventions based on the implementation of those guidelines provide a framework for more active participation of teachers and students to self-assessment and solution of specific problems.

Keywords: interdisciplinary curriculum, guidelines, interdisciplinarity, problem centered curriculum and teaching practicum.

\section{Introducción}

Distintas voces reconocen la importancia de la práctica docente para el desarrollo profesional de los futuros maestros (Gimeno, 2007; Davini, 2001; Tsui, 2003; Gutiérrez, 1996; Wallace, 1991; Borg, 2004; Richards y Lockhart, 1994). La naturaleza teórico-práctica de los saberes privilegiados en los modelos pedagógicos de formación docente discuten la necesaria relación entre saberes disciplinares, prácticos, experienciales y reflexivos, a fin de acometer la compleja tarea de formar docentes críticos y propositivos, capaces de proponer innovaciones. Ante este escenario, algunos programas adoptan propuestas curriculares de corte integral e interdisciplinario, como una condición de posibilidad que establezca puentes entre saberes, articule la relación entre teoría y práctica y permita intervenciones pedagógicas transformadoras. La LEBEI (Licenciatura en Educación Básica con énfasis en Inglés) es uno de esos casos. A pesar de que el programa posee documentos que sustentan la naturaleza problémica de su metodología de trabajo (LEBEI, 1998; 1999; 2004; 2006; 2010; 2011) al indagar en su población estudiantil, se pone de manifiesto que no reconoce este elemento como un eje vertebral, ni del discurso ni del trabajo docente. Más aún, algunos docentes admitieron tener dificultades para abordar el trabajo interdisciplinario.

Los propósitos misionales y de formación declarados en el currículo oficial presentan dificultad para articularse en la práctica. Del currículo oficial al currículo práctico (Posner, 2004) se advierten una serie de desafíos que se desatan de la diversa interpretación conceptual y metodológica de las partes. Esta revisión suministra valiosa información sobre cómo se articula la relación entre teoríapráctica y la forma en la que los profesores, estudiantes, académicos y administrativos asumen dicha articulación. A raíz de las diversas perspectivas en la forma en la que se asume la práctica docente en las escuelas, la variable apropiación metodológica de los estudiantes, los conflictos que surgen en la dirección de la práctica, las inconformidades de algunos directivos dentro y fuera del programa, entre otros, se emprendió un diagnóstico con base en la resolución de cinco grupos de preguntas alrededor del origen del currículo, sus propósitos y contenidos, así como de su funcionamiento y de la percepción que se tiene del mismo.

En este marco se buscó analizar: 1. Los procesos instructivos y la realidad de la práctica desde una perspectiva que les dota de contenido (revisión de syllabus en términos de núcleos problémicos, ${ }^{4}$ objetivos y procesos); 2. La intersección de prácticas diversas que se refieren a los procesos de tipo pedagógico, interacciones y comunicaciones educativas (observación de prácticas, revisión de planeadores de clase, diarios de campo y proyectos pedagógicos); y, 3. El discurso sobre la interacción entre la teoría y la práctica (mediante la revisión de programas y documentos del programa) (Gimeno, 2007). Desde esta perspectiva, la investigación permitió identificar, por un lado, un grupo de desafíos alrededor de las prácticas docentes y los actores involucrados en la misma y, por otro, el origen de unas necesidades que debían considerarse a la luz de unos lineamientos interdisciplinares. Entre los

4 Núcleos problémicos es el nombre usado para describir las áreas de acción de trabajo de las diferentes asignaturas en el currículo del programa en el que se desarrolló este proyecto. 
principales desafíos se destacaron: ausencia de interdisciplinariedad en los espacios académicos relacionados con la práctica docente, falta de solidez y profundidad teórica y, sobretodo, carencia de práctica en los espacios académicos de metodología.

Particularmente se registró inconsistencia de un modelo de acompañamiento a los estudiantes por parte de los directores de práctica. Surgen en contexto unas necesidades que enuncian un mayor empoderamiento del Proyecto Educativo del Programa, la mayor apropiación del concepto de interdisciplinariedad y la metodología problémica. Adicionalmente, se determina la relevancia del fortalecimiento de los horizontes de formación, la articulación de la práctica con la investigación y una mayor fundamentación de la formación pedagógica integral e interdisciplinaria que se evidencie en los espacios prácticos.

Este trabajo de investigación se centró en una orientación teórica y metodológica de corte cooperativo para acompañar el proceso de diseño, implementación y evaluación de actividades y lineamientos interdisciplinarios, con miras al fortalecimiento de la práctica docente. Inicialmente se exponen algunos postulados teóricos frente a la relación teoría-práctica en currículos de corte problémico e interdisciplinario. Acto seguido, se describen y detallan las fases de investigaciónacción que sirvieron como base para el diseño, implementación y evaluación de los lineamientos y se presentan los resultados. Finalmente, se incluyen las conclusiones, a partir de la implementación de los lineamientos y algunas recomendaciones para este tipo de diseño.

\section{Consideraciones teóricas}

\section{Intervenciones curriculares en educación superior}

Las intervenciones curriculares en educación superior presentan diversas propuestas. Por ejemplo: el currículo asumido como proyecto o plan educativo pretendido o real (Inciarte y Canquiz, 2011; González, 2010), como expresión formal y material del proyecto (Gairín, 1995), como campo práctico en el que se intersectan prácticas diversas (Gimeno, 2007) y como vínculo entre teoría y práctica en educación (Gil, 2009). Apuestas que se mueven en dos tendencias claramente identificables: las que tratan al currículo como algo estático, objetivo y aprehensible; y las que advierten en él relaciones dinámicas, complejas e interactivas. En esta investigación la segunda tendencia aportó mayores luces para asumirlo como una entidad que cambia, se alimenta de experiencias, prácticas, éxitos y fracasos.

El eje central de dicha revisión retomó aquellos estudios centrados en la formación docente (Davini, 2001; UNESCO, 2006); las prácticas como escenarios de reflexión e integración (TovarGálvez, y García, 2012; Perrenoud, 2001); y la interdisciplinariedad como enfoque metodológico para solucionar problemas específicos y promover la participación y la reflexión de los actores involucrados en el proceso de enseñanza y aprendizaje (Martinello y Cook, 2000).

En términos generales se identificaron algunos aspectos sustanciales alrededor de: 1 . La preocupación por la orientación curricular; 2. La falta de articulación entre teoría y práctica; y 3 . La evaluación comoejeparala transformación curricular. Este acercamiento orientó un esquema cíclico para las fases de diseño, evaluación e implementación de lineamientos, mientras se revisaban elementos de teoría curricular cruciales para el programa de formación docente: práctica pedagógica, currículo problémico e interdisciplinariedad.

\section{La práctica pedagógica y el desarrollo curricular problémico}

Es importante que toda propuesta de formación docente apunte hacia la construcción de sujetos críticos y reflexivos, capaces de proponer alternativas e innovaciones pedagógicas que contribuyan a comprender mejor la escuela y su problemática. Se trata, en suma, de superar las tradiciones netamente "eficiencistas, academicistas y disciplinadoras" (Davini, 2000, p. 57), en aras de configurar el trabajo pedagógico, no como un mero proceso adaptativo, sino como un proceso de ampliación 
de la conciencia y la emancipación. De acuerdo con Grundy (1987), el trabajo de práctica debe entenderse como una praxis que se apoya en los siguientes principios: a) tiene que ser una práctica sustentada por la reflexión dentro de un proceso circular que comprende la planificación, la acción y la evaluación (investigación-acción); b) ser contextualizado; c) ocuparse de interacciones sociales y culturales; e d) incluir conflictos.

Cuando profesores y estudiantes no apropian una apuesta como praxis en sus metodologías y didácticas específicas, uno de los riesgos del currículo estriba en la incapacidad de los usuarios de reconocer críticamente las fallas en el nivel práctico. Es urgente, entonces, que los proyectos educativos y los modelos pedagógicos que se sustentan en las licenciaturas resignifiquen la relación teoríapráctica, desde una perspectiva investigativa. Según Carr y Kemmis (1988, p. 128): "hace de la práctica una cosa más teórica, en el sentido de enriquecerla mediante la reflexión crítica, sin que al mismo tiempo deje de ser práctica por cuanto ayuda a formular más concluyentemente los juicios que informan la práctica investigativa”. Desde esta perspectiva, es necesario repensar la educación teniendo en cuenta la investigación que motive la reflexión crítica. En palabras de Palos (2000) "la reinterpretación ética del currículo". Es decir, redimensionando ese concepto de formación humana integral y sus implicaciones en los desarrollos pedagógicos de los distintos actores.

Autores como Davini (2001), Tsui (2003), Gutiérrez (1996), Wallace (1991), Borg (2004) o Richards and Lockhart (1994), entre otros, consideran relevante quelasinstituciones encargadas de la formación de docentes resignifiquen la forma en la que se articulan los saberes, con el propósito de coadyuvar en la construcción de conocimiento reflexivo de corte integrador. Dicha reflexión facilitaría que un docente esté en condiciones de modificar su práctica, en forma consciente y creativa y, en esa medida, adquiera una capacidad de analizarla críticamente (Vera, 1985). Desde aquí se puede colegir la necesidad de que los programas de formación docente integren en su propuesta curricular lineamientos de índole interdisciplinaria e integral. Un docente formador de formadores podría así orientar el proceso de articulación de las prácticas emergentes de esta fase de formación profesional, a la biografía escolar previa de sus estudiantes $\mathrm{y}$, posteriormente, a su futuro ejercicio docente (Davini, 2001).

Para el propósito anterior, se hace necesario articular el conocimiento experiencial de los estudiantes con el conocimiento de la institución formadora. De esta manera, el estudiante reconocerá que sus reflexiones enriquecen su formación pedagógica y resignifican su rol como futuro docente. Tal proceso constituye la forma en la que se negocia la información proveniente de la teoría y de la práctica que ponen de manifiesto funciones cognitivas de orden superior como la abstracción, la extrapolación y la inferencia para asir conclusiones. Partimos del supuesto que todo intento por mejorar aspectos de formación docente está íntimamente ligado a la estructura curricular. De este modo, la articulación reflexiva de los estudiantes conllevaría a evidenciar la consolidación curricular.

Cuando la relación teoría-práctica se encuentra fundamentada en un currículo problémico, que pretende ser integral e interdisciplinario, evidenciar estos procesos de consolidación curricular requiere una mirada más aguda. En el caso particular de la LEBEI, los acometidos misionales se orientan a la formación de un docente investigador e innovador capaz de transformar las realidades del contexto (LEBEI, 1999). Esto implica una mirada un tanto precavida frente a varios aspectos: a las formas en la que se trabaja al interior de las aulas; a los roles de docentes y estudiantes; a la funcionalidad y pertinencia del modelo pedagógico, los espacios y tiempos dedicados a la integración disciplinar; al grado de apropiación y reconocimiento de la apuesta metodológica; entre otros. Todo esto con el fin de determinar cómo existe correspondencia entre el currículo ideal y el práctico.

Para el análisis de este proyecto, acuñamos el enfoque del eclecticismo reflexivo de Posner (2005), que sugiere dar cuenta de las ventajas y desventajas del currículo haciendo posible advertir los peligros que acechan al currículo, las oportunidades de aprovechamiento, las ventajas y las posibilidades de minimización de las desventajas curriculares. 
Desde este enfoque, el currículo se erige como el escenario de convergencia de prácticas diversas que resultan de la apropiación de ideales, consensos y disensos sobre el qué y cómo enseñar y sobre el qué y cómo aprender. En este sentido, la relevancia de la relación teoría-práctica es indudablemente una razón fundamental para actualizar la discusión en torno a los problemas curriculares que, a la vez, son agentes en la configuración de las prácticas de enseñanza (Gimeno, 2007, p. 32). Para el caso de un currículo problémico, el diseño curricular debe ir más allá de la formulación de contenidos fijos y respuestas absolutas, en cuanto a que los núcleos problémicos se postulan con la intención de tratar de cuestionar la realidad, comprenderla profundamente, de pensar y repensar realidades, investigar las posibles realidades, colocarlas a prueba, formular hipótesis e implementar procesos de cambio. Su inserción en el plan de estudios debe estar sustentada por una dinámica conceptual de desarrollo flexible e integral que implique la organización de secuencias de conocimientos en núcleos problémicos y ejes temáticos (Lederman, 1992 citado en Doc. de Vicerrectoría, (UDFJC); pero siempre con el objetivo de articular la realidad social con la realidad institucional.

La puesta en acción de un currículo problémico implica el accionar en las prácticas de enseñanzaaprendizaje de un dialogismo problémico (Skatkin, 1971), en el que se privilegia el ejercicio indagativo y reflexivo frente a la generación de conocimiento situado. Por consiguiente, el conocimiento se problematiza, se sitúa, se construye y se deconstruye para dar paso a una transformación de los estados de conciencia y aproximación al conocimiento desde distintas perspectivas. Así pues, los docentes $\mathrm{y}$ estudiantes asumen el conocimiento como inacabado y adoptan diferentes lecturas para decantar las intersubjetividades que le dan sentido a las realidades curriculares.

\section{Interdisciplinariedad}

La interdisciplinariedad es un fenómeno de convergencia de disciplinas que se complementan para dar explicaciones a problemáticas cuya naturaleza no puede asirse desde una sola mirada. El individuo es el encargado de reconocer, describir y analizar dichas conexiones. La integralidad no existiría si el pensamiento humano no interviene para establecer esas conexiones. En este sentido, compartimos con Resweber (1981) la idea de que la interdisciplinariedad es una actitud del sujeto por comprender una problemática, atendiendo a la naturaleza compleja de la misma a través de su indagación.

La metodología que orienta un currículo interdisciplinario se centra, de esta manera, en la indagación. Martinello y Cook (2000) la definen como el procedimiento por el cual se guía al estudiante a examinar, explorar, ahondar, inquirir, averiguar, preguntar, sondear, investigar, escudriñar, buscar, escrutar, interrogar y estudiar para llegar al conocimiento y la verdad. Dichos procesos se constituyen en características primarias de la interdisciplinariedad que obligan a establecer relaciones y conexiones para la solución de una problemática. El poder de la indagación interdisciplinaria reside en su capacidad de estimular una diversidad de formas de pensamiento y así, aumentar las posibilidades de efectuar conexiones creativas e "ir más allá de la información dada" (Bruner, 1973, p. 69).

\section{Lineamientos interdisciplinarios}

Al revisar los documentos del programa se advierte una ruptura epistemológica en la forma de concebir la relación teoría-práctica y las formas particulares de trabajo, a partir de núcleos problémicos y temáticos. Desde la investigación se acudió a la literatura especializada en la construcción de conocimiento vía dicha metodología (Brant, 1991; Beane, 2005; Davis, 1999; Gatewood, 2002; Palmer, 1991). El ejercicio ayudó a señalar, como diría Popper (1972, p. 95), que "somos estudiosos de problemas, no de disciplinas"; y, por tanto, la mirada interdisciplinaria implica una metodología, un concepto, un estilo de pensamiento y una ideología reflexiva.

Como parte del proceso, hubo un consenso generalizado sobre la necesidad de contar con ejes transversales para acceder a la interdisciplinariedad y a la reinterpretación ética del currículo, a fin de hacer posible el encuentro de soluciones a problemáticas desde diferentes perspectivas. Tales ejes hacen ineludible establecer los parámetros 
que darían origen a esa reinterpretación. Es así como nuestra propuesta parte de estipular unos "lineamientos" que sean la guía de trabajo que fortalezca cualquier clase de construcción del conocimiento del individuo. Dichos lineamientos no constituyen un listado de estrategias para seguir al pie de la letra, al contrario, son enunciados que guían la ruta a seguir para llegar al pensamiento crítico que vislumbre las conexiones entre disciplinas y, por tanto, las diferentes perspectivas de los problemas. Se puede hacer un paralelo con la definición de estándares que según Cassassus (citado en Palos, 2000) son informaciones para ser utilizadas como referencia, que se sitúan en el ámbito de la acción, son sistematizadas por personas autorizadas, nos permiten actuar con mayor seguridad, nos informan acerca de lo que se espera sea el resultado del proceso, producto o servicio que queremos utilizar, deben estar disponibles públicamente, indican y delimitan responsabilidades.

Así pues, los lineamientos interdisciplinares no pueden ser solamente el listado de unos parámetros fijos e inmodificables para guiar el actuar de los personajes involucrados en su ejecución. Por el contrario, estas guías deben enmarcar la conjunción de saberes que vigorizan ese actuar. En consecuencia, los lineamientos deben proporcionar criterios, orientaciones, una delimitación de responsabilidades que de cara al público generen una concientización de los objetivos enmarcados por cada programa. Los lineamientos también deberían tener un componente de flexibilidad que posibilite la intervención individual, ya sea de los estudiantes, del maestro o cualquier miembro de la comunidad que conforma el contexto con todas sus características individuales y colectivas.

\section{Metodología}

La investigación se inscribe en el modelo mixto (Mc Millan, 2008), con una metodología de investigación acción (Kemmis y Tagar, 1988; Nunan, 1989) que toma como base el enfoque de métodos mixtos, cualitativamente orientados (Morse y Neihaus, 2009), donde se busca integrar elementos cuantitativos y cualitativos durante la indagación teórica y los datos empíricos de los ciclos referentes a la investigación acción. Los elementos se integran a la fase de diseño, implementación y evaluación de lineamientos curriculares interdisciplinarios. Se trata de un enfoque que busca entender la complejidad y los contextos de experiencia social y educativa para resolver un problema. Para este caso, la ruptura entre lo teórico-práctico en las relaciones interdisciplinarias en el espacio académico de prácticas docentes.

Los resultados obtenidos de distintos instrumentos (encuestas, entrevistas, tablas de frecuencia), fuentes (documentales, testimoniales, grupos de trabajo) y materiales (documentos de trabajo, documentos oficiales, grabaciones), durante las distintas fases de la investigación, condujo a un proceso de triangulación mediante un tipo de análisis de contenido dominantemente cualitativo. La apuesta buscó identificar los elementos recurrentes a través de una tabla de frecuencia de aquellos aspectos que profesores, estudiantes y administrativos vinculaban con dificultades $\mathrm{y}$ fortalezas. Lo que permitió la identificación de dos categorías para pensar los ejes de articulación interdisciplinaria: 1. la formación docente, en la cual se identificaron los aspectos de índole epistemológico y conceptual alrededor de la formación de docentes de inglés, revisando los conceptos defendidos desde la propuesta curricular de interdisciplinariedad y modelo pedagógico de formación y práctica docente; y 2. las prácticas docentes, en la que específicamente se analizó su conceptualización, la ubicación del espacio académico en el plan de estudios, las disposiciones para la búsqueda de colegios, su articulación con otros espacios del plan de estudios y su impacto hacia la proyección social que postula la universidad y el proyecto curricular en su misión.

El seguimiento de dichas categorías permitió que en el diseño cíclico de la investigación, las fases se dieran en ocasiones de manera simultánea, por ejemplo, la revisión documental y evaluaciones de actividades realizadas en distintos momentos y con diferente población. Dicha forma se refleja en el desarrollo analítico realizado en este artículo.

El siguiente esquema ilustra la articulación de las fases de investigación: 


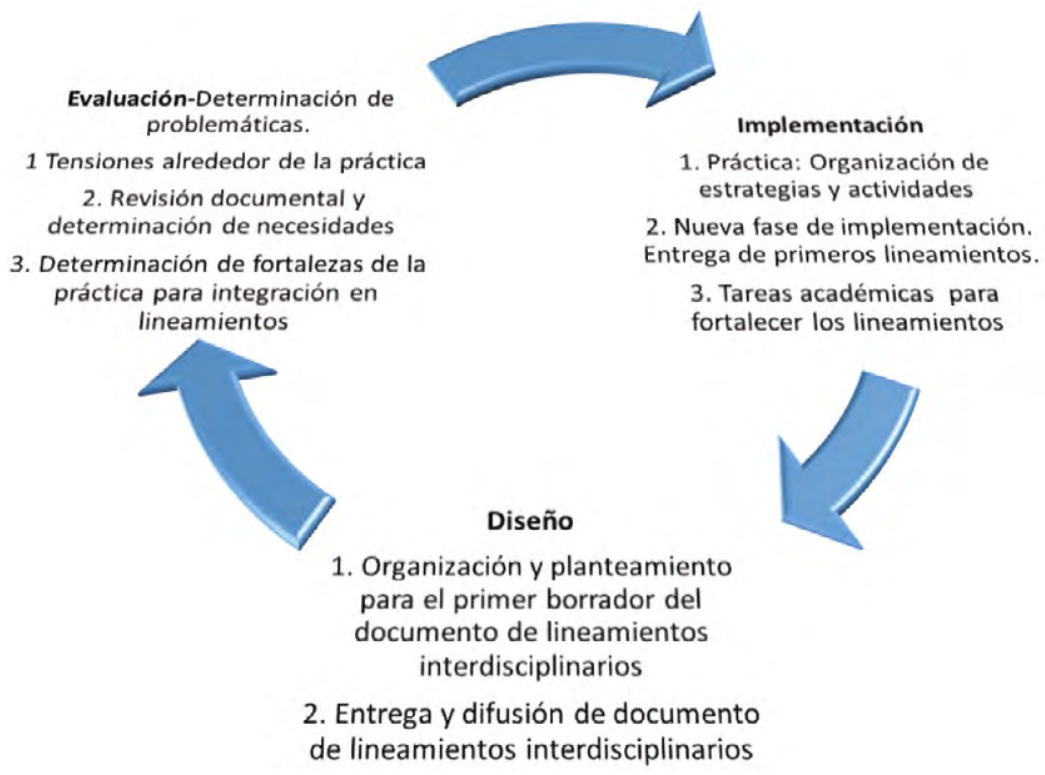

Figura. 1. Fases de la investigación. Fuente: elaboración propia.

Para la etapa de análisis de datos, el procedimiento se fundamentó en lo siguiente:

Estudio documental de textos escritos tales como: Documento de Acreditación Previa del programa (2000), Documento de Acreditación de Alta Calidad (2004-2006), Reglamento de Prácticas Docentes (S.f.), programas de clase y demás documentos oficiales de la LEBEI (Actas e informes de autoevaluación, 2004).

Estudio y muestreo de datos y documentos testimoniales, obtenidos de encuestas, actas y talleres.

Análisis-síntesis e inducción deducción, en la recopilación y procesamiento de la información y en la articulación de los elementos que permitieron la comprensión de las orientaciones para la elaboración de los lineamientos interdisciplinarios. Tipo de análisis que favorece la exploración de un campo de estudio para encontrar orientaciones de las cuales derivar posibles intervenciones. Aquí es posible el diseño de tablas de frecuencia, soporte estadístico, muestreo para establecer comparaciones de frecuencia de aparición de los elementos retenidos como unidades de información o de significación: palabras, partes de frases, frases enteras, etc. (Pinto y Grawitz, 1967). De esta manera se cumplieron las siguientes tareas:

1. Identificación de los criterios teóricos que sustentan el modelo pedagógico de formación de la Licenciatura en Educación Básica con Énfasis en Inglés.

2. Comprensión de los criterios que tiene la licenciatura acerca de la práctica y su disposición en el plan de estudios.

3. Reconocimiento de las fortalezas y debilidades del modelo de mentoría de prácticas docentes.

4. Definición del concepto de interdisciplinariedad que subyace en la propuesta de formación docente de la LEBEI.

5. Articulación de actividades y orientaciones para la implementación de lineamientos interdisciplinarios.

6. Para el diseño, la evaluación e implementación de los lineamientos se tuvieron en cuenta los aspectosdeteoríacurriculardefinidosporGimeno (2007) y Palos (2000); frente a la necesidad de intensificar temas de corte transversal las propuestas de López (2004) y Martinello y 
Cook (2000); para el empoderamiento de la indagación interdisciplinaria y la integralidad curricular, Beane (2005), entre otros. Para el caso específico de las orientaciones de prácticas docentes se integraron elementos conceptuales de Davini (2001), Tsui (2003), Gutiérrez (1996), Wallace (1991), Borg (2004) y Richards y Lockhart (1994), ante la necesidad de articular los saberes experienciales y de formación institucional para hacer de la práctica un espacio reflexivo y de indagación.

\section{Resultados y discusión}

\section{La fase evaluación-diagnóstica}

En esta etapa se tuvo en cuenta la valoración de la formación docente, la interdisciplinariedad y las prácticas docentes por parte de docentes y estudiantes (Palos, 2000). ${ }^{5}$ Dicha fase arrojó información acerca de la incidencia del plan de estudios en formación integral de los estudiantes como futuros docentes, la incidencia de las asignaturas en el desempeño de las prácticas docentes, la preparación previa de los estudiantes para enfrentarse a la vida de la escuela, las dificultades frente a su quehacer docente en las escuelas donde se llevó a cabo la práctica, la presencia o ausencia de la interdisciplinariedad en las diversas asignaturas y la articulación entre los programas propuestos por los docentes y las orientaciones del Proyecto Curricular. El estudio de las valoraciones encontradas nos permite identificar las siguientes tensiones:

La preparación para la práctica necesita ser fortalecida interdisciplinariamente por otros espacios académicos

Por un lado, los estudiantes muestran claridad sobre la interdisciplinariedad de los espacios académicos, para fortalecer los procedimientos metodológicos propios del ejercicio de la profesión. Esto reveló la necesidad de analizar los diferentes niveles de apropiación del concepto de interdisciplinariedad que tienen los maestros en sus prácticas, en relación con la formación integral y la díada teoría-práctica. Aunque los profesores afirmaron trabajar con un enfoque interdisciplinario, se les dificultó identificar ejemplos concretos

5 Encuestas aplicadas a 260 personas, entre estudiantes (237) y docentes (23). de cómo orientaban esta articulación con otros espacios académicos, por lo cual consideraron necesario que el proyecto curricular aporte lineamientos para fortalecer la interdisciplinariedad, la integralidad y la práctica docente. Por otro lado, se evidenció que los estudiantes reconocen otras problemáticas, como la necesidad de acercarse al contexto real con anterioridad a su práctica, ya que en la realidad dicho acercamiento teórico-práctico es insuficiente.

Esta situación pone de manifiesto que es necesario ir más allá de enunciar un enfoque de trabajo para que exista un conocimiento preciso sobre cómo abordarlo, sobre qué instancias metodológicas deben emprenderse y qué tipo de estrategias son las más adecuadas para analizar problemas. A pesar de que se enuncian los temas y núcleos problémicos en el currículo, aparentemente esto no garantiza el trabajo problémico e interdisciplinario.

La intensidad horaria de las metodologías para la enseñanza del inglés y sus contenidos requieren más articulación

En la LEBEI, existe un espacio académico que complementa las prácticas docentes llamado metodologías. Dichos espacios tienen una intensidad horaria de dos horas y se toman simultáneamente a las prácticas pedagógicas. Tanto estudiantes como profesores señalan que la intensidad horaria y la ubicación de estas metodologías en el plan de estudios no contribuyen plenamente para el desarrollo de las prácticas por varias razones: 1 . Se presenta desfase de núcleos temáticos; 2. Hay dificultad para abordar distintos contextos prácticos; y 3. Hay una idealización de situaciones alejadas de la realidad de los colegios. Dicha situación no favorece el desarrollo teórico-práctico que se desliga de la relación entre metodologías y espacios de práctica, ya que la complejidad, las inquietudes y problemas que tienen los estudiantes en su práctica docente son múltiples y espontáneos. Adicionalmente, expresan que otros espacios académicos como Diseño de Materiales del campo pedagógico no ocupan un lugar estratégico en la malla curricular en correlación a la práctica. Serían mejor aprovechados si éstos fueran trasladado a semestres anteriores a la práctica. A pesar de que hubo un ajuste curricular 
que permitió aumentar las horas y anticipar los espacios de metodología a los de práctica docente, los contenidos y formas de trabajo privilegiadas (exposiciones, y lecturas, etc.) no parecían favorecer una genuina orientación de lo que sucede en los colegios de práctica, ni de la variedad de dificultades e intereses de los estudiantes.

En consecuencia, el problema ya no es de intensidad horaria o ubicación en el plan de estudios, sino de las dificultades que subyacen alrededor de cómo orientar la relación teoría-práctica, que va más allá de los contenidos del syllabus, las lecturas, los productos, etc. Se trata de advertir en la complejidad del evento pedagógico, la potencia para intervenir interdisciplinariamente la realidad.

Las distintas interpretaciones al acompañamiento que realizan los directores de práctica revelan dificultades para una comprensión consensuada del modelo pedagógico de práctica docente

Los estudiantes afirmaron que los directores de práctica docente hacen un acompañamiento adecuado, sin embargo señalaron que las diferencias individuales y metodológicas (tiempo de permanencia en el colegio, socializaciones y reuniones conjuntas para la discusión de experiencias) entre docentes resultan cruciales para lograr un mejor aprovechamiento de los espacios de discusión, reflexión y observación. Indicaron que algunos de sus directores de práctica observan sus clases completas, mientras que otros, se enfocan en la retroalimentación de los distintos momentos de desarrollo de la misma e identifican las dificultades y las fortalezas de los estudiantes para enriqueciendo las discusiones y las evaluaciones.

En consecuencia, se evalúa que se requiere que el director de prácticas independientemente de su metodología, se interese por conocer la escuela, que además tenga experiencia pedagógica e investigativa en la enseñanza del inglés en primaria y secundaria, así como el hecho de que tenga capacidades para liderar procesos académicoinvestigativos, de manera que pueda pensar su propio acompañamiento. De esta forma, que promueva la indagación y la interdisciplinariedad conjugando los elementos del ambiente y su experticia profesional.
Fase de implementación-práctica. Previo y paralelo a lineamientos interdisciplinares

Consiste en la implementación de estrategias metodológicas y divulgación para consolidar un plan de mejoramiento. Por lo tanto, la identificación de las anteriores tensiones conllevó al equipo de investigadores a emprender un trabajo de socialización de dichos resultados a través de la realización de talleres y reuniones de trabajo con profesores y algunos estudiantes. En esta segunda fase, sobresalieron actividades de reflexión y actualización curricular, tales como: taller sobre integralidad curricular, revisión y actualización de núcleos problémicos del plan de estudios, y de igual manera, actividades de diseño e implementación como: diseño de núcleos interdisciplinares, diseño de un encuentro interdisciplinar y lineamientos para la consolidación del Proyecto Educativo del programa.

Como resultado de esta fase se afianzaron varios documentos que la LEBEI articuló a uno solo denominado Proyecto Educativo del Programa. Este documento no existía en físico, sino que estaba disperso en la memoria colectiva de los profesores más antiguos y algunas grabaciones de reuniones. Entre ellos, Integralidad curricular en la LEBEI (2011), Historia del Proyecto Curricular LEBEI (1998) y Naturaleza problémica del programa.

Desde esta investigación no se pretendió reducir el problema a una cuestión de teoría curricular, ni tampoco se ambiciona una mejor organización estructural de la práctica, a fin de que el plan de estudios solucionara la problemática. Aquí planteamos la necesidad de un reposicionamiento y empoderamiento de los roles que tienen los usuarios del currículo, desde la postura ética a la que se refiere Palos (2000), para el logro de los objetivos de formación integral e interdisciplinaria. Así pues, este proceso se daría mediante la participación democrática de la población por medio de las actividades descritas anteriormente. Sin embargo, para que este desarrollo tenga mayor solidez, la documentación es parte vital. La diferencia radica en que la construcción de un documento no significa el seguimiento literal de parámetros (Palos, 2000), sino al contrario, es una oportunidad para recoger las voces de maestros y alumnos y consolidar 
aspectos de la vida real del ejercicio docente en una fundamentación teórica dinamizante.

Esta acción concentrará otro conjunto de acciones de una forma coherente y consensuada, que consecuentemente apuntará a la transformación pedagógica de los futuros docentes en una formación más integral.

\section{Fase de evaluación}

En esta fase se llevó a cabo el análisis documental de los postulados del modelo pedagógico de formación y prácticas docentes, instaurado en los documentos oficiales del programa: 1. Se revisó el enfoque epistemológico de la LEBEI, que está ligado a la heurística del enfoque problémico; 2. Se analizó el énfasis que se le otorga a las asignaturas referentes a la práctica que señalan la articulación de modelos reflexivos e investigativos; 3. Se examinó el énfasis en la proporción de asignaturas en inglés, como apuesta a mejores desempeños en esta lengua; 4. Se definió el rol que desempeñan los distintos campos de formación para la formación integral del estudiante en aras de identificar la articulación interdisciplinaria, alrededor de la pedagogía como núcleo fundante de la formación; 5. Se señaló la definición de práctica docente que sustenta la propuesta, precisando su carácter reflexivo y la articulación con la dispuesta en el reglamento de prácticas; y 6. Se determinó la presencia o ausencia del enfoque interdisciplinario en los programas de clase.

El cruce de estos hallazgos con los de la primera fase de evaluación permitieron identificar dos puntos neurálgicos, a saber: la teoría y la práctica necesitan de mayor articulación frente a los conceptos de interdisciplinariedad e integralidad curricular; y la definición de prácticas docentes necesita una fundamentación más coherente con el modelo pedagógico del programa en torno a la reflexión, la investigación y el sentido crítico. De esta manera, se debe superar la definición instrumental del reglamento que la circunscribe a un espacio para poner en práctica los conocimientos previamente adquiridos. Frente a la revisión de los hallazgos de estas fases se precisan los siguientes retos:
Empoderamiento del proyecto educativo del programa

El conocimiento que docentes y estudiantes tienen del modelo pedagógico del programa es fragmentado, este aspecto posiblemente se debe a la inexistencia de un documento unificado que condense los pilares del mismo. Por lo tanto, desde esta investigación se contribuyó en la compilación y edición del Proyecto Educativo del Programa, para promover su divulgación entre docentes y estudiantes en el que pudiera tener acceso en cualquier momento. Este documento fue publicado por la Universidad Distrital Francisco José de Caldas en el año 2012 y es de acostumbrada distribución y trabajo en las jornadas de inducción a estudiantes nuevos. No obstante, su existencia no garantiza el empoderamiento si los usuarios del currículo no se reconocen en este proyecto educativo.

\section{Mayor apropiación delconcepto interdisciplinariedad y de la metodología problémica}

Los programas de clase revisados en la fase de evaluación no revelan explícitamente el uso de la palabra interdisciplinariedad en alguno de sus componentes (introducción, metodología, justificación, contenidos o evaluación). Las bibliografías propuestas en su mayoría eran de corte disciplinar — situadas desde los saberes particulares - y los núcleos problémicos, en un alto porcentaje, no coincidían con los dispuestos en el documento de núcleos problémicos del programa. Por lo tanto, se hizo urgente crear mecanismos de difusión de los acuerdos establecidos respecto a hacer consciencia en la comunidad sobre la relevancia de la interdisciplinariedad en el desarrollo integral de nuestros estudiantes del programa. Entre estos mecanismos se destacan: un apartado en los programas de clase, donde los profesores explicaran cómo ese espacio que dirige aborda la interdisciplinariedad, bibliografías, cibergrafías, etc.; que destaquen textos procedentes de distintas áreas de conocimiento, reuniones de trabajo por ciclos de formación, es decir en el eje vertical del plan de estudios.

\section{Fortalecimiento de los horizontes de formación}

El aporte a la formación pedagógica no se hace evidente en todos los programas de clase, a pesar de que el documento de acreditación previa la 
señala como núcleo fundante. El perfil de formación del docente de inglés está orientado hacia la consolidación de un docente investigador, usuario competente de lengua inglesa y analista y didacta de la misma, entre otros aspectos. De este modo, todos los docentes de nuestra licenciatura deben enriquecer sus prácticas en pro de la diferenciación de un programa encargado de formar docentes y uno enfocado solamente en competencias comunicativas en el idioma, que lo diferencie con cualquier otro profesional. Esto supone establecer la distinción entre un usuario y un pedagogo de la lengua extranjera. Lo que significa el despliegue de niveles de formación que garanticen que el futuro docente aprenda inglés para enseñarlo, valorando elementos del contexto, el currículo de las escuelas, las resistencias, etc. Lo anterior supone la promoción de una serie de actividades para:

- Generar orientaciones metodológicas de corte interdisciplinar que articulen de mejor manera el desarrollo de las prácticas docentes del programa con los problemas de las escuelas y colegios distritales.

- Propiciar reflexiones pedagógicas integrales e integradoras de las actividades propias del quehacer pedagógico de los distintos actores del currículo.

- Impulsar la sistematización de experiencias y la formalización de protocolos de seguimiento a los diversos momentos de la práctica.

- Generar espacios de participación activa de todos los participantes del currículo, pero principalmente de los docentes adscritos al programa.

\section{Articulación de la práctica con la investigación}

En el documento de acreditación previa se explica el modelo pedagógico de práctica docente imbricado en la reflexión y la articulación de saberes previos y de saberes de formación profesional. A pesar de que en el perfil se apuesta por un docente investigador, no se fundamenta cómo se articula la investigación en la práctica docente. Este hecho denota una necesidad de trabajar en este aspecto interdisciplinario. Adicionalmente, existe un reglamento de prácticas del programa que se presenta como constitutivo de la misma, precisamente como elemento de índole operativa, su existencia regula las interacciones de los participantes en ese evento pero no parece subyacer como un elemento de índole conceptual. Se requiere así una mayor fundamentación a la luz de la teoría curricular provista para los programas de formación docente, de las necesidades de formación integral del practicante y de los niños y jóvenes de los colegios a la luz de la interdisciplinariedad y la integralidad.

En la reglamentación de la práctica se estipulan objetivos, deberes y requisitos, entre otros aspectos de los cuales se infiere una marcada tendencia a enfocarse en la didáctica del inglés instrumentalizada, una explícita descripción del espacio académico como un lugar de aplicación de conocimientos teóricos, metodológicos y psicopedagógicos, un énfasis en la resolución de problemas propios del área de inglés, una tendencia a asumir al coordinador de prácticas como observador y corrector. En este sentido, se intuye un paradigma de formación docente que privilegia la disciplinariedad y marca la especialidad por encima de otros componentes que se proponen en el mismo currículo. Se ubica como objeto de investigación a la población beneficiada en relación con el aprendizaje del inglés como enfoque principal. Pocas veces se denota que se haga con la práctica misma, la escuela o el docente practicante, es decir, un favorecimiento del fenómeno intra áulico.

Por lo tanto, se hace necesario fundamentar la articulación de la práctica con la investigación en los sujetos, objetos y procesos constitutivos de la misma. Se trata de una nueva dirección epistemológica que abra las posibilidades de interacción entre focos de análisis para el trabajo pedagógico y para los docentes de distintas áreas de conocimiento.

\section{Mayor fundamentación de la formación pedagógica integral e interdisciplinaria y su evidencia en la práctica}

La fundamentación teórica de la interdisciplinariedad y la formación integral en los documentos del programa es amplia y, en términos generales, arroja información que deja inferir elementos de aplicación. No obstante, ejemplos concretos de la aplicabilidad y de cómo aporta a la formación integral de los estudiantes son indispensables para la comunidad. En consecuencia, se procuró que el diseño de los lineamientos interdisciplinarios proveyera una de esas formas 
concretas posibles de trabajo, de manera tal que los profesores las percibieran como orientaciones concretas de su trabajo. Una de estas formas, consistió en establecer relaciones entre núcleos temáticos ${ }^{6}$ y problemas de los espacios académicos de un semestre.

Identificada la preocupación que englobaba a los núcleos y a los temas, a cada descriptor del perfil se le buscó un ejercicio de mejoramiento. Para el caso, el ser docente, la apuesta consistiría en analizar el estado del arte sobre la cuestión para analizar los discursos subsidiarios provenientes de ciencias interesadas en el tema y luego emprender un proceso dialéctico. De esta manera, se valoraron los aportes de cada una de ellas y se buscó articular un discurso sólido con perspectiva interdisciplinaria (Méndez, 2013).

Docentes comprometidos con la formación integral de sus estudiantes reclaman sujetos capaces de superar el sincretismo pedagógico de la especialidad para afrontar los distintos fenómenos que influyen en la escuela y propiciar escenarios de inclusión. Así pues, sugirieron pensar los problemas de la niñez y la juventud. Desde esta perspectiva, se hizo necesario apostarle al reto de formar un docente de inglés que cuestione su propia práctica y, se piense a sí mismo como un agente de cambios que propone alternativas de solución a las distintas problemáticas de la niñez, la juventud, la escuela y la sociedad.

En los lineamientos se propone superar la limitada y superficial comprensión de la práctica docente como un espacio de aplicación de conocimientos previamente adquiridos, en el que se verifican teorías, métodos y materiales —desligada de la teorización y la construcción de nuevo conocimiento-, para comprenderla como un espacio de meta-teorización, en el que la indivisible relación teoría-práctica se resignifique; propiciando cambios sustanciales en sus actores y haciendo posible la generación de nuevo conocimiento a través de procesos de investigación.

Las orientaciones para el mejoramiento de la práctica docente obligan a atender los distintos

6 Nombre usado para describir las grandes áreas donde se enuncian los núcleos problémicos. aspectos de la teoría curricular, que se precisan en los distintos programas de formación docente (Lafrancesco, 2003; Palos, 2000; López, 2004; Stenhouse, 1975). En este último sentido, la práctica debe admitir elementos que posibiliten que los participantes del currículo contribuyan desde distintas disciplinas y que esto genere interrelaciones para comprender el contexto. De esta manera, los procesos de enseñanza aprendizaje propician la identificación más explícita de la articulación entre teoría y práctica (Mann, 2003; Gervains y Correa, 2004; Ayala, 2005; Wallace, 1991 y Tsui, 2003).

Volviendo a la mirada ética que enuncia Palos (2000), en el compromiso de formación en la universidad, se exhorta a los docentes formadores de docentes a hacer una reinterpretación ética de su propia práctica a la luz de los acometidos de formación integral, que se exigen desde un currículo problémico. Interrogantes tales como ¿Estimulo la formación investigativa de mis estudiantes? ¿Explicito y ejemplifico las distintas relaciones disciplinarias que convergen en el saber a mi cargo? ¿Favorecen las metodologías y las didácticas que privilegio distintas formas de comprender los problemas y de contextualizarlos? ¿Es mi discurso, un discurso de empoderamiento de la propuesta curricular? ¿Oriento de manera dialógica las inquietudes de mis estudiantes? ¿Problematizo y hago referencia directa constante a los problemas que nos ocupan?; entre muchos otros interrogantes, conforman formas concretas que permanentemente guían la práctica y la forma de orientar a los estudiantes. Tal ejercicio incentiva la indagación y, por lo tanto, promueve la interdisciplinariedad para el análisis concreto de problemáticas de la vida real.

\section{Fase de diseño}

En esta fase se realizó un primer borrador del futuro documento Lineamientos interdisciplinarios. Como se dijo anteriormente, este documento sería un documento de trabajo, un marco referencial para el trabajo continuo y reformulación de objetivos del currículo a la luz de los principios fundantes del mismo. En el marco de este trabajo de lineamientos se diseñó un taller para los directores de práctica y se diseñó un instrumento de evaluación que se aplicó a los estudiantes. Dichos instrumentos buscaron 
determinar la forma como cada espacio académico favorecía el desarrollo de la interdisciplinariedad. Adicionalmente, se indagó a los estudiantes sobre los aspectos que consideraban fortalezas y debilidades del trabajo pedagógico orientado por los docentes. Este punto ayudaría a encontrar elementos que hubiesen fortalecido las prácticas por medio de los talleres y actividades de interdisciplinariedad.

\section{Nueva fase de implementación}

Esta segunda fase de implementación inició en el primer semestre del 2008. Se buscaba entregar a los profesores del proyecto curricular un avance del documento Lineamientos interdisciplinarios para fortalecer las prácticas docentes (ver Anexo). A partir de ese momento, se continuó un trabajo más amplio de compilación y actualización de documentos fruto de las orientaciones de los lineamientos interdisciplinares. Algunos elementos surgieron como necesarios para la actualización de procedimientos que favorecieran la interdisciplinariedad del programa. Entre ellos:

- Modificación de formatos de programas de clase, articulando aspectos específicos que evidenciaran las conexiones interdisciplinarias del espacio académico. Se buscó también concretarlos en los aspectos de perfil del estudiante que se fortalece en el programa.

- Actualización de los núcleos problémicos del plan de estudios.

- Identificación de puntos críticos del currículo. Consecuentemente se buscó realizar una fundamentación más precisa de los campos de formación. ${ }^{7}$ Aspectos que se recogen en un documento actualizado de Proyecto Educativo del Programa (2011) y del documento Reflexiones sobre integralidad (2010).

- Implementación de los Encuentros interdisciplinarios. Actividad propuesta para fortalecer el trabajo interdisciplinario en el ciclo de fundamentación, ${ }^{8}$ el cual obtuvo la

7 Los Campos de Formación son espacios de la malla curricular que le dan sentido al perfil profesional. Por ejemplo, un campo de formación es el investigativo (para más información, ver Proyecto Educativo del Programa, 2010).

8 El currículo del programa LEBEI se encuentra dividido en ciclos, los primeros semestres se denominan ciclo de fundamentación. Este ciclo precede las prácticas docentes. más baja valoración de la interdisciplinariedad en la información recogida y por lo tanto que necesitaba atención inmediata.

Por otro lado, algunos aspectos positivos del modelo de prácticas docentes se integraron a la propuesta de lineamientos, atendiendo las características propias del documento. Entre estos aspectos sobresalieron:

- Modelo de acompañamiento y orientación de algunos directores de práctica que privilegian la reflexión, la indagación, la investigación y la escritura. A su vez, sustentadas y variadas formas de trabajo que privilegian las discusiones, seminarios, debates, mesas redondas, etc.

- Criterios más coherentes para la selección de colegios para la realización de la práctica ligados a la misión de la Universidad y a los intereses investigativos de profesores y estudiantes.

- La fundamentación teórica de los elementos curriculares tales como integralidad, interdisciplinariedad y metodología problémica. A la vez que se incorporaran bibliografías actualizadas; libros, artículos e informes de investigación.

- Definición operativa de deberes de los practicantes, los directores de práctica, los profesores titulares y los colegios a la luz del modelo integral del programa y de sus intereses interdisciplinares de indagación.

- Definición de aspectos de la evaluación por proceso que dieran cuenta de la evolución del estudiante.

En el proceso el grupo de investigadoras también piloteó un instrumento de seguimiento a procesos académicos que permitiera realizar un análisis de la percepción que tenían los estudiantes sobre interdisciplinariedad, a partir de las actividades dispuestas vía lineamientos. Dicho instrumento no solo fue diseñado para contribuir a la mirada interdisciplinaria objetivo de esta investigación, sino que, a su vez, se presentó como un aporte para analizar al unísono otros factores que sirvieran de base para mejorar procesos académicos al interior del programa. Entendiendo la interdisciplinariedad como la conjunción de disciplinas para solucionar una problemática. En 
un cuestionario diseñado como instrumento de seguimiento se incluyó una pregunta que apuntaba a reconocer la forma en que la interdisciplinariedad se veía entre los diferentes espacios académicos. Dicha pregunta jugó un papel de vital importancia en esta investigación, para visualizar el impacto del currículo en la población estudiantil al igual que su forma de concebirlo.
Los 150 estudiantes encuestados (entre VI y VIII semestre) afirmaron haber encontrado mayor interdisciplinariedad en los espacios correspondientes a los campos investigativo y pedagógico. Esto ocurrió en un rango que oscila entre un $65 \%$ a un $90 \%$. Entre los aspectos destacados sobresalen la complementariedad (36\%) y la pertinencia (25\%) (gráfico 1).

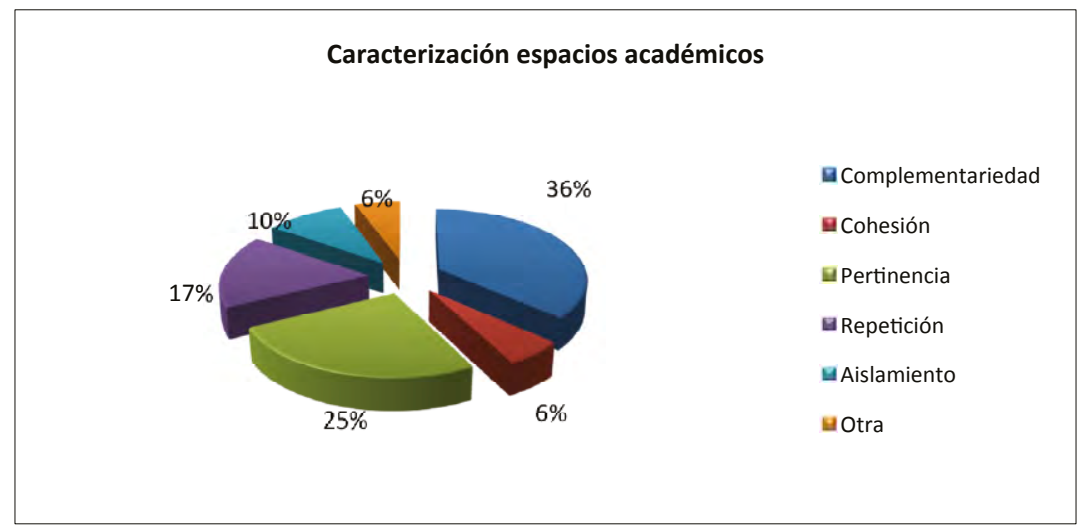

Gráfico 1. Fortalezas del ciclo de profundización. Fuente: elaboración propia.

Como puede colegirse de los anteriores resultados, la percepción sobre la interdisciplinariedad denota que los estudiantes encontraron mayor apropiación sobre la interdisciplinariedad, y que sus percepciones podrían no solamente identificar sino también clasificar la forma en que se daba la relación interdisciplinaria. A juicio de las investigadoras, esto surge como fruto de las distintas actividades de trabajo, socialización de los lineamientos y determinación de los puntos críticos del currículo que se identificaron a través de la investigación.

\section{Conclusiones}

El proceso de esta investigación llevó a cabo una comprensión reflexiva del currículo y de las percepciones de sus usuarios, para posibilitar pensar la crítica como una oportunidad de mejoramiento e identificación de los desafíos que conllevan la pérdida de reconocimiento de los horizontes de formación del proyecto educativo del programa (2010). En este caso un currículo problémico interdisciplinario que se debilita en la práctica porque se desliga del análisis complejo de la realidad, a partir de sus propios principios.

Acciones tales como: grupos de trabajo, elaboración de documentos, discusiones, revisiones y actualizaciones del currículo, enmarcadas en una serie de lineamientos interdisciplinarios contribuyeron a una considerable mejoría de procesos, como da cuenta la percepción de los estudiantes y los docentes. Lo que demuestra, por un lado, que hubo una mayor apropiación de las condiciones que plantea este enfoque de trabajo y, por otro lado, que el proceso de intervención curricular puede ser exitoso en la medida en que las distintas instancias, directivos, profesores y estudiantes se involucren para autoevaluar y autoevaluarse. Así es posible propiciar intervenciones a corto, mediano y largo plazo. 
Los lineamientos interdisciplinares se propusieron en este proyecto como orientaciones generales que encontraron al espacio de prácticas docentes como un elemento transversal en el cual todos los campos de formación tienen parte. Como documento de orientación, no fue diseñado como un recetario de actividades, sino como un documento de trabajo que requiere de aportes, críticas y reformulaciones de docentes y estudiantes. No obstante, más allá del documento de lineamientos, las actividades de reflexión, discusión y empoderamiento del discurso pedagógico fueron los que hicieron visible algunos ejemplos concretos de teoría curricular. Entre ellos, integralidad, interdisciplinariedad, transdisciplinariedad, solución de problemas en las formas de trabajo y acompañamiento docente. Vale la pena adicionar que esto ocurrió no solo en las prácticas sino en otros espacios académicos.

\section{Referencias}

Ayala, J. (2005). Beyond reflections in and out of the classroom (Tesis de Grado). Licenciatura en Educación Básica con Énfasis en Inglés. Bogotá D.C.: Universidad Distrital Francisco José de Caldas.

Beane, J. (2005). La integración del curriculum. Madrid: Morata.

Borg, M. (2004). Key concepts in ELT the apprenticeship of observation. ELT journal, 58(3), 274-276. Recuperado de: http://eltj.oxfordjournals.org/cgi/ reprint/58/3/274.pdf

Brant, R. (1991). On interdisciplinary curriculum: A conversation with Heidi Hayes Jacobs. Educational Leadership, 49(2), 24-26.

Bruner, J. (1973). Going Beyond the Information Given. New York: Norton.

Carr, W. y Kemmis, S. (1988). Teoría crítica de la enseñanza. La investigación acción en la formación del profesorado. Barcelona: Martínez Roca.

Davini, M. (2001). La formación docente en cuestión: política y pedagogía. Buenos Aires: Paidós.

Davis, M. (1999). Design's inherent interdisciplinarity: The arts in integrated curricula. Arts Education Policy Review, 101(1), 8-13.
Gairín, J. (1995). El Proyecto educativo y el desarrollo del currículo. Aula de Innovación Educativa, 1(38), 6-12.

Gatewood, T. (2002). Interdisciplinary curriculum planning process. Recuperado de http://www.vmsa. org/vmcent/links.html

Gervains, C. y Correa, M. (2004). Explicitación del saber de experiencia de los profesores en el contexto de las prácticas docentes: Un marco conceptual y metodológico. Íkala, Revista de Lenguaje y Cultura, 9(15) 141-167.

Gil, R. (2009). La dimensión social del curriculum: Una visión desde Nicaragua. Buenos Aires: LPP, Laboratorio de Políticas Públicas.

Gimeno, J. (2007). El currículo: una reflexión sobre la práctica. Madrid: Morata.

Grundy, S. (1987). Curriculum: Product of praxis. Londres: The Farmel Press.

González, B. (2010). El Currículo como proyecto educativo en sus tres niveles de concreción. Recuperado de http://www.curricular.info/visiones/ documentos/gonzalez.pdf

Gutiérrez, G. (1996). Student foreign language teacher's knowledge growth. In D. Freeman and J.C. Richards (eds), 50-78.

Inciarte, A. y Canquiz, L. (2011). Análisis de la consistencia interna del currículo. Informe de Investigaciones Educativas, 15(1-2), 2-9.

Kemmis, S. y McTaggart, R. (1988). Cómo planificar la investigación-acción. Madrid: Laertes.

Lafrancesco, G. (2003). Nuevos fundamentos para la transformación curricular. Bogotá D.C.: Magisterio.

LEBEI. (1998). Historia del Proyecto Curricular LEBEI. Bogotá D.C.: Universidad Distrital Francisco José de Caldas.

LEBEI. (1999). Documento de Acreditación Previa. Bogotá D.C.: Proyecto Curricular LEBEI. Universidad Distrital Francisco José de Caldas.

LEBEI. (2004). Documento de Autoevaluación y Acreditación del Programa. Bogotá D.C.: Proyecto Curricular LEBEI. Universidad Distrital Francisco José de Caldas.

LEBEI. (2006). Documento de Créditos Académicos y Flexibilidad Curricular. Bogotá D.C.: Proyecto Curricular LEBEI. Universidad Distrital Francisco José de Caldas.

LEBEI. (S.f.). Reglamento de Prácticas Docentes. Bogotá D.C.: Proyecto Curricular LEBEI. Bogotá D.C.: Universidad Distrital Francisco José de Caldas. 
LEBEI. (2010). Proyecto Educativo del Programa de LEBEI. Bogotá D.C. Universidad Distrital Francisco José de Caldas. Publicación interna del programa LEBEI.

LEBEI. (2011). Reflexiones sobre integralidad. Bogotá D.C.: Universidad Francisco José De Caldas. Publicación interna del programa LEBEI

López Jiménez, N. (2004). Retos para la construcción curricular. Colombia: Mesa redonda. Magisterio.

Mc Millan, J. (2008). Educational research. Fundamentals for the consumers. London: Pearson Education.

Mann, S. (2003). Alternatives perspectives on professional practice in academia development. In H. Eggins \& R. Macdonald. (Eds). The Scholarship of Academic Development. Buckingham: Opening University Press. 80-90

Martinello, M L. y Cook, G. (2000). Indagación interdisciplinaria en la enseñanza y el aprendizaje. Barcelona: Gedisa.

Méndez, P. (2013). Currículo en la formación docente. Colombia: Fondo de Publicaciones de la Universidad Distrital

Morse, J. M., E Niehaus, L. (2009). Mixed method design: Principles and procedures (Vol. 4). Left Coast Pr.

Nunan. (1989). Designing tasks for the communicative classroom. UK: Cambridge University Press.

Palmer, J. (1991). Planning wheels turn curriculum around. Educational Leadership, 49(2), 57-59.

Palos, J. (coord.) (2000), Estrategias para el desarrollo de los temas transversales del currículum, Barcelona, Horsori (Cuadernos de Educación núm. 31).

Perrenoud, Ph. (2001). La formación de los docentes en el Siglo XXI. Revista de Tecnología Educativa, 14(3), 503-523.

Pinto, R. \& Grawitz, M. (1967). Analyse de contenu et theorie. En Méthodes des sciences sociales (pp. 456-499). Paris: Dalloz.
Popper, K. (1972). Conjeturas y refutaciones. Barcelona: Paidós. [Orig. 1963].

Posner, G. (2005). Análisis de currículum. México: McGraw-Hill.

Resweber, J. (1981). El método interdisciplinario. París: Universidad de Francia.

Richards, J. E Lockhart C. (1994). Reflective teaching in second language classroom. Cambridge: Cambridge University Press.

Skatkin, M. (1971). Perfeccionamiento del proceso de enseñanza. Moscú: Pedagogía.

Stenhouse, L. (1975). An introduction to Curriculum Research and Development, London: Heinemann.

Torres, J. (2006). Globalización e interdisciplinariedad: El currículo integrado. Madrid: Morata.

Tovar-Gálvez, J. y García, G. (2012). Investigación en la práctica docente universitaria: obstáculos epistemológicos y alternativas desde la Didáctica General Constructivista. Educ. Pesqui. 38(04), p. 881-895.

Tsui, A. (2003). Understanding expertise in teaching. Case study of ESL teachers. Cambridge: Cambridge University Press.

Universidad Distrital Francisco José de Caldas. (1992). Flexibilidad y créditos académico. Documento de Vicerrectoría. Bogotá D.C.: Autor.

UNESCO. (2006). Modelos innovadores en la formación inicial docente. Recuperado de http://unesdoc. unesco.org/images/0014/001465/146544s.pdf

Vera, R. (1985). Orientaciones básicas de los talleres de educación. Santiago de Chile: Paidós.

Wallace, M. (1991). Training foreign language teachers. Cambridge: Cambridge University Press. 
Diseño, implementación y evaluación de prácticas pedagógicas

\section{Anexo \\ Lineamientos interdisciplinarios para fortalecer la práctica docente}

\section{Descripción de la propuesta}

Esta propuesta nace del interés general del equipo de profesores vinculados a la dirección de las prácticas docentes de la Licenciatura en Educación Básica con énfasis en Inglés, y como fruto de diferentes reflexiones, inquietudes e investigaciones realizadas a lo largo del tiempo; recoge una serie de orientaciones generales con el propósito de mejorar el desarrollo de las prácticas docentes, que los estudiantes de la Licenciatura en Educación Básica con énfasis en Inglés realizan en varias instituciones de básica primaria y secundaria en el Distrito Capital.

Estas orientaciones se ubican en un paradigma más amplio de actuación, supeditado al marco filosóficoepistemológico del currículo problémico, integrado e interdisciplinar que sirve de plataforma pedagógica al programa. En este sentido, no pretende ser un plan riguroso de clase a seguir, sino más bien un documento de consulta abierto a la crítica, a la complementación, a la adecuación, etc., de los potenciales usuarios del mismo.

\section{Objetivos de la propuesta}

Este documento de lineamientos interdisciplinares y actividades académicas se propone:

- Generar orientaciones metodológicas de corte interdisciplinar para mejorar el desarrollo de las prácticas docentes del programa de Licenciatura en Educación Básica con énfasis en Inglés de la Universidad Distrital Francisco José de Caldas.

- Servir de base para reflexiones pedagógicas integrales e integradoras de las actividades propias del quehacer pedagógico de los distintos actores del currículo LEBEI.

- Impulsar la sistematización de experiencias y la formalización de protocolos de seguimiento a los momentos de la práctica.

- Generar espacios de participación activa de todos los usuarios del currículo, pero principalmente de los docentes adscritos al programa.

Impulsar el desarrollo de la identidad docente

El docente en formación debe legitimar su rol desde el discurso mismo que lo articula como miembro de una comunidad educativa y social, y construir un proceso argumentativo frente a su quehacer, definiendo su ideología y ética para comprender que sus compromisos y acometidos profesionales trascienden y resignifican los contractuales, en la búsqueda de una educación más integral, reflexiva e interdisciplinaria.

\section{La formación pedagógica}

El trabajo pedagógico forma parte de un complejo proceso de evolución de los sujetos que exhorta al futuro docente a hacer una proyección de sí mismo y a formarse un autoconcepto de su propia imagen como profesor. Este autoconcepto hace parte del "querer ser" que, en la mayoría de las veces, se forma de un ideal extraído de la educación profesional (adquirida en instituciones encargadas de formar pedagogos); de la formación práctica (adquirida en las prácticas profesionales); y de su experiencia escolar previa (adquirida en los modelos de su formación escolar). Esta última fase, la de la biografía escolar de los estudiantes, de acuerdo con Davini (2001, p. 80), es una fase previa que se constituye en un trasfondo de saber del paso por la cultura de la escuela. 


\section{La interdisciplinariedad}

Las líneas metodológicas y métodos transversales tienen que partir de la particularidad de las instituciones que tienen sus propios objetivos y finalidades. En este orden de ideas, Palos (2001) señala que una reinterpretación ética de los mismos pone de manifiesto la urgencia de establecer dinámicas de trabajo de los distintos actores de la comunidad educativa que posibiliten un desarrollo más integral y disciplinario.

Teóricamente esto resulta fácil de probar atendiendo la teoría curricular defendida desde este tipo de propuesta, pero en la práctica se requiere de diseños curriculares flexibles y de equipos de trabajo dispuestos a coadyuvar este proceso.

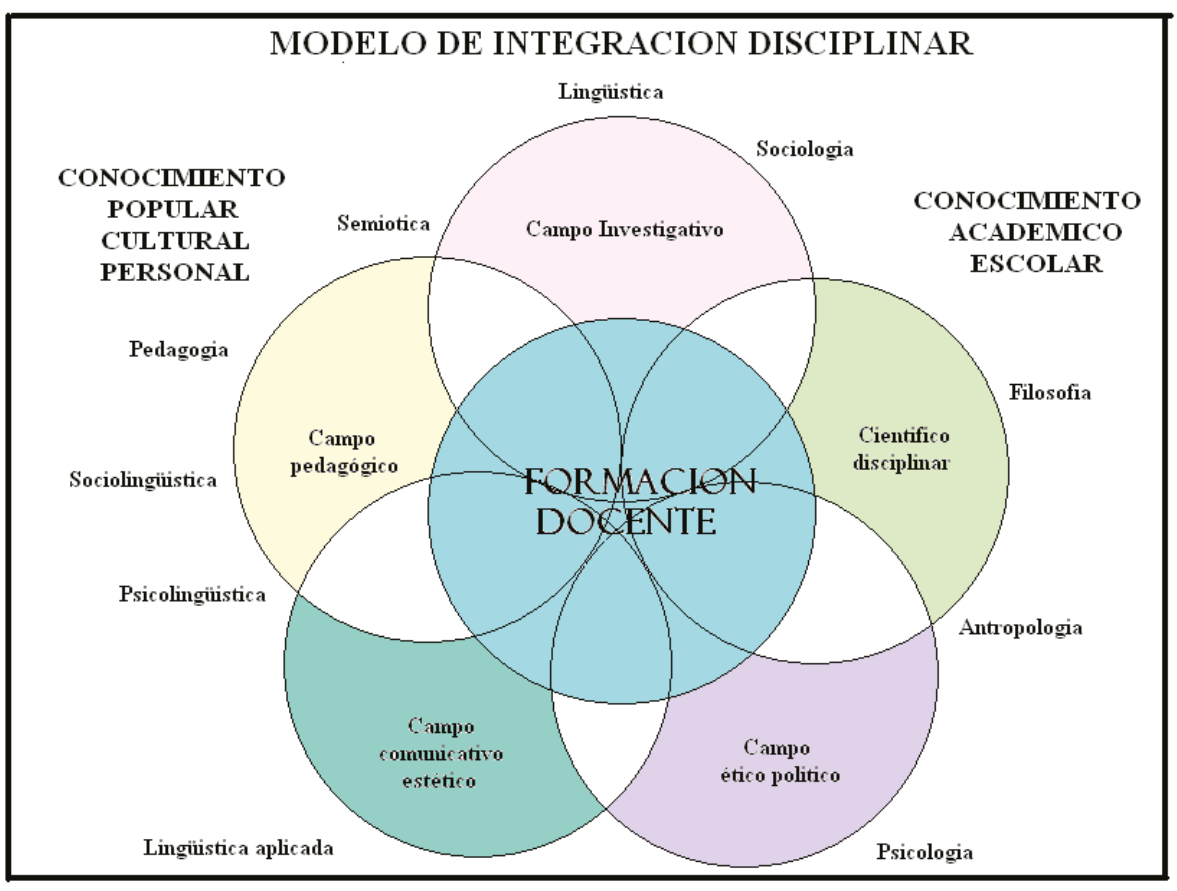

Figura 2. Modelo de integración disciplinar.

Fuente: elaboración propia.

Ahora bien, la organización de unos saberes dentro de un proyecto curricular, como es el caso de los programas de formación de la Facultad de Ciencias y Educación de la Universidad Distrital Francisco José de Caldas, plantea dinámicas de renovación e innovación pedagógica. El proyecto curricular, según Gimeno (2007, p. 16) "sirve para traducir nuevas ideas sobre lo que deben ser los estudios sociales, la ciencia, junto a planteamientos adecuados sobre cómo aprenden los estudiantes y cómo debe operar metodológicamente el profesor, etc.".

En este sentido, los proyectos curriculares son una manera de vertebrar las distintas áreas de conocimiento y experiencia para hacer realidad otras concepciones educativas, acordes con los argumentos a favor de una formación docente y educativa más integral (Torres, 2006).

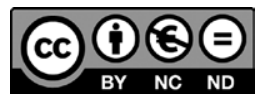

